J Neurosurg 119:825-827, 2013

(C)AANS, 2013

\title{
Erratum
}

\section{Toll-like receptor 4 expression during cerebral aneurysm formation}

\author{
Laboratory investigation
}

To The EDItor: We appreciate the publication of our paper entitled "Toll-like receptor 4 expression during cerebral aneurysm formation. Laboratory investigation" $(J$ Neurosurg 113:851-858, 2010). Since publication, some errors in this paper have been identified, and we would like to correct the record at this time. Specifically, we made an error in representing control data as well as in providing proper attribution to journals in which some figure panels were previously published. The errors do not make any changes in the findings of our study.

The following corrections have been made to our article:

1. A paragraph has been added to the end of the subsection Immunohistochemical Studies in the Methods section. The addition is as follows:

Several tissue slices were obtained from one experimental animal. One of these slices was treated with elastic van Gieson staining for a reference image of immunohistochemical studies. We have used this same image of elastic van Gieson-stained tissue in immunohistochemical studies of other molecules. ${ }^{7}$

2. Two sentences were added to the end of the paragraph in the subsection Western Blotting in the Methods section. The sentences are:

We examined some molecules using the same membrane by stripping a previously used antibody. We then used the same blotting image of the internal control, $\alpha$-tubulin, for Western blot analyses of other molecules. ${ }^{4}$

3. In Expression of TLR4 During Cerebral Aneurysm Formation, in the Results section, we found two errors in the second paragraph. The corrected text now reads as follows (corrections bolded):

Please include this information when citing this paper: published online July 5, 2013; DOI: 10.3171/2013.6.JNS09329a.
In Western blotting, expression of TLR4 was significantly upregulated 1 month after aneurysm induction (5 data sets, $p$ $<0.01)$. Three months after aneurysm induction, TLR4 expression decreased to the level before aneurysm induction ( 5 data sets, 1 month compared with 3 months; $\mathrm{p}=0.021$ ) (Fig. $3 \mathrm{~A}$ and $\mathrm{B}$ ).

4. Portions of Figs. 2 and 3 have been replaced. In addition, credit has been given to two other journals in which some figures first appeared, specifically:

Figure 2A first appeared as Supplemental Figure 1A in Aoki T, Kataoka H, Ishibashi R, Nozaki K, Morishita R, Hashimoto N: Reduced collagen biosynthesis is the hallmark of cerebral aneurysm: Contribution of interleukin-1b and nuclear factor-kB. Arterioscler Thromb Vasc Biol 29:1080-1086, 2009. This article is now credited in the legend to Fig. 2 and can be found in the updated reference list.

Figure $2 \mathrm{C}$ has been replaced because the original was incorrectly placed. The new Fig. 2C first appeared as Figure $1 \mathrm{~A}$ in Aoki T, Kataoka H, Morimoto M, Nozaki K, Hashimoto $\mathrm{N}$ : Macrophage-derived matrix metalloproteinase-2 and -9 promote the progression of cerebral aneurysms in rats. Stroke $38: 162-169,2007$. This article is now credited in the legend to Fig. 2.

Figure 3 has been replaced because a portion of it was labeled incorrectly. This figure was modified from Fig. 1I in Aoki T, Kataoka H, Ishibashi R, Nozaki K, Egashira K, Hashimoto N: Impact of monocyte chemoattractant protein-1 deficiency on cerebral aneurysm formation. Stroke 40:942-951, 2009. This article is now credited in the legend to Fig. 2 and can be found in the updated reference list.

5. The reference list has been updated to accommodate two additional articles specified in Point 4.

This article contains some figures that are displayed in color online but in black-and-white in the print edition. 
Erratum

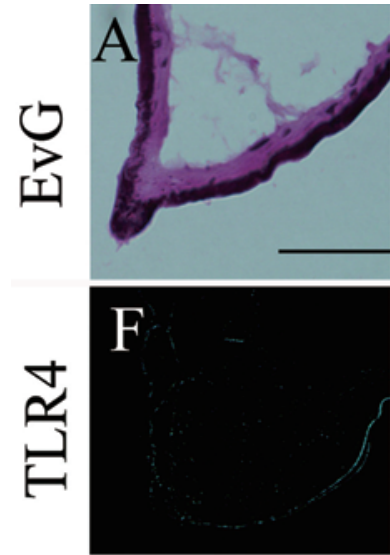

$0 \mathrm{M}$
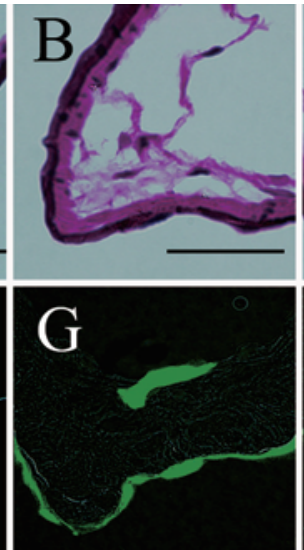

$0.5 \mathrm{M}$
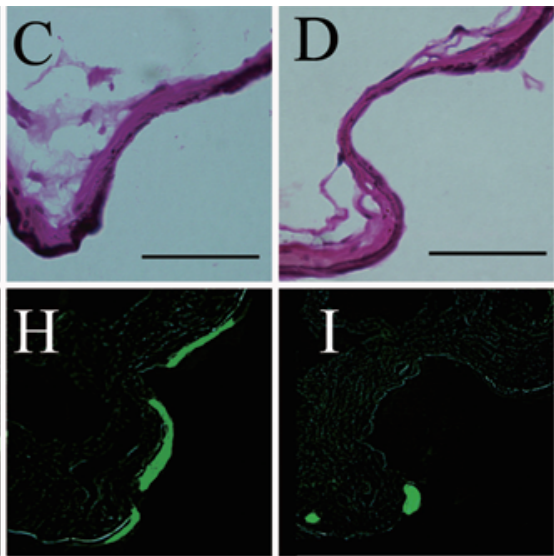

$1 \mathrm{M}$

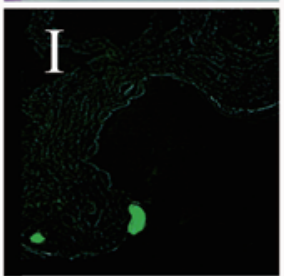

$3 \mathrm{M}$

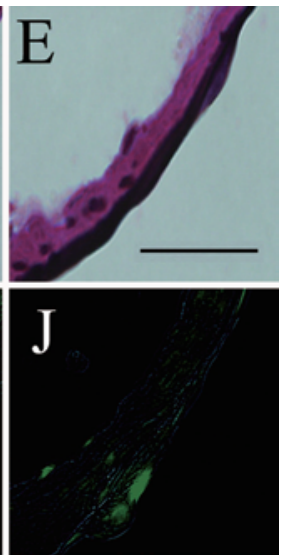

1M:contra

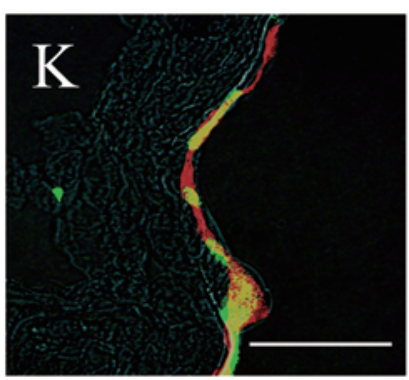

eNOS/TLR4

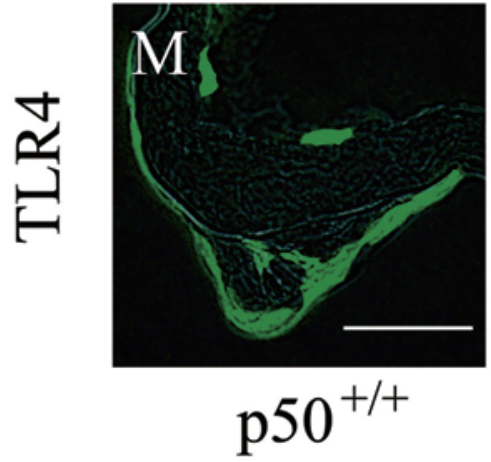

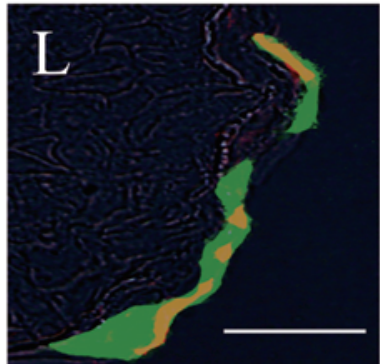

ap65/TLR4

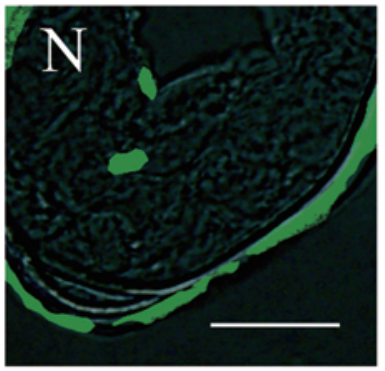

$\mathrm{p} 50^{-/-}$

FIG. 2. Photomicrographs showing expression of TLR4 in cerebral aneurysm wall and NF- $\kappa B$ activation. A-E: Elastica van Gieson (EvG) staining of the serial sections next to panels $F(A), G(B), H(C), I(D)$, and J (E). F-J: Immunostained sections showing expression of TLR4 in cerebral aneurysm walls at the following time points: before (F), 2 weeks after (G), 1 month after $(\mathrm{H}), 3$ months after $(\mathrm{I})$, or at the contralateral ACA-OA bifurcation 1 month after $(\mathrm{J})$ aneurysm induction. K: Double immunostaining of endothelial cell marker (eNOS: red color) and TLR4 (green color) 1 month after aneurysm induction. L: Double immunostaining of the DNA-binding form of NF-KB (ap65: red color) and TLR4 (green color) 1 month after aneurysm induction. $\mathbf{M}$ and $\mathbf{N}$ : Immunostaining of TLR4 in cerebral aneurysm walls of wild-type $\left(\mathrm{M}, \mathrm{p} 50^{+++}\right)$and $\mathrm{NF}-\mathrm{\kappa} B \mathrm{p50}-\mathrm{deficient}(\mathrm{N}$, $\mathrm{p} 50^{--}$) mice 5 months after aneurysm induction. Bars $=50 \mu \mathrm{m}(\mathrm{A}-\mathrm{J})$ and $20 \mu \mathrm{m}(\mathrm{K}-\mathrm{N}) .3 \mathrm{M}=3$ months after aneurysm induction; $1 \mathrm{M}$ :contra $=$ contralateral ACA-OA bifurcation at 1 month after aneurysm induction. Panel $\mathrm{A}$ is reproduced with permission from Aoki et al: Arterioscler Thromb Vasc Biol 29:1080-1086, 2009. Panel C is reproduced with permission from Aoki et al: Stroke 38:162-169, 2007.

We apologize to the editor and readers, and we are pleased to have the opportunity to make these corrections. The errors were corrected online as of July 5, 2013.

Masaki Nishimura, M.D., Ph.D.

Kyoto University Graduate School of Medicine

Kyoto, Japan 
Erratum
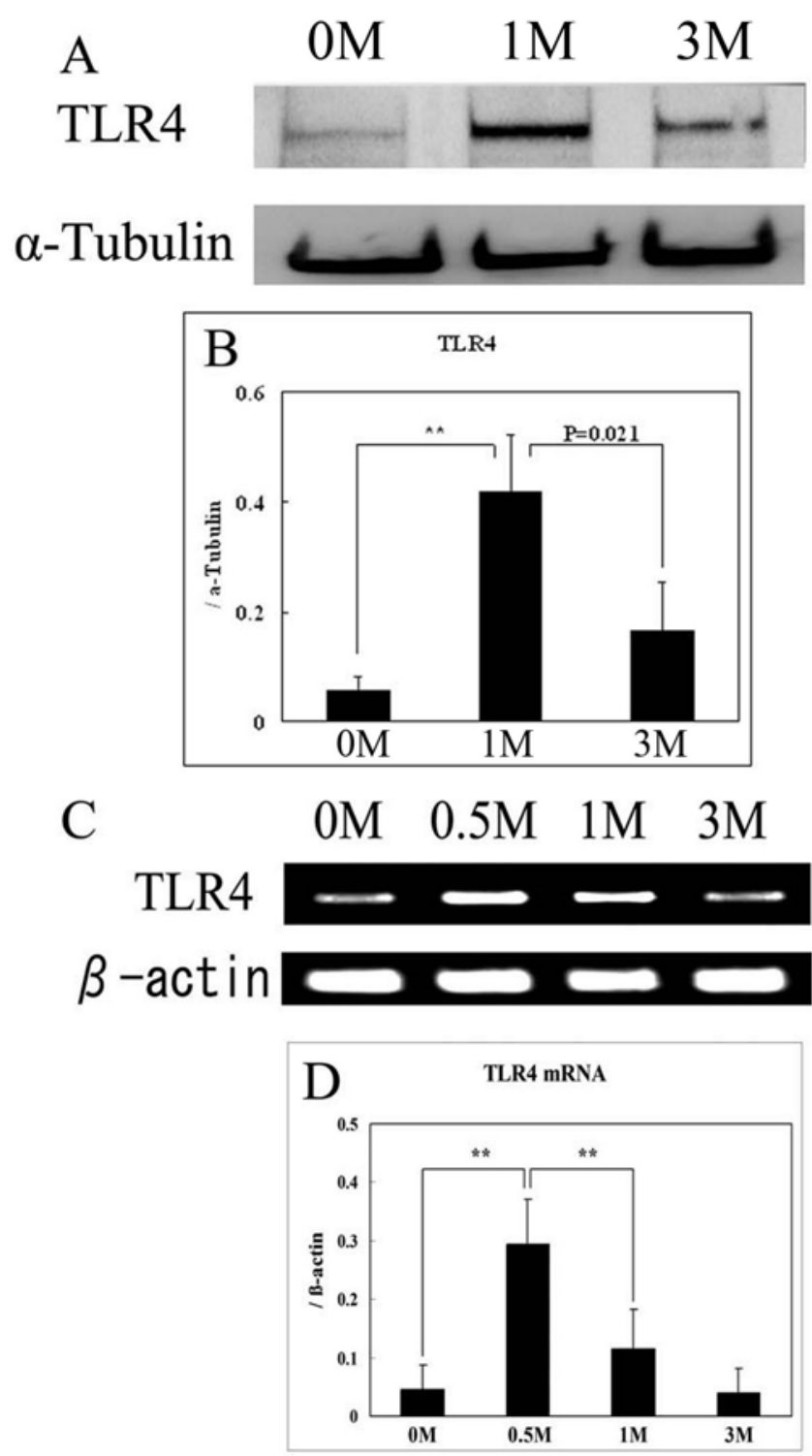

Fig. 3. Protein and mRNA expression of TLR4 in cerebral aneurysm walls of rats. A: Western blotting of TLR4. Representative data from 5 independent experiments are shown; $\alpha$-tubulin served as an internal control. B: Densitometric analysis of Western blotting in 5 data sets. C: Results of RT-PCR studies of TLR4 in cerebral aneurysm walls. Representative data from 6 independent experiments are shown; $\beta$-actin was used as an internal control. D: Densitometric analysis of RT-PCR in 6 data sets. Double asterisks indicate $p<0.01$. Panels A and $B$ are reproduced with permission from Aoki et al: Stroke 40:942-951, 2009. 